九州大学学術情報リポジトリ

Kyushu University Institutional Repository

\title{
DYADIC STATIONARY PROCESSES AND THEIR SPECTRAL REPRESENTATIONS
}

Nagai, Takeaki

Faculty of Engineering, $0 i$ ta University

https://doi.org/10.5109/13115

出版情報：統計数理研究. 17 (3/4)，pp.65-73，1977-03. Research Association of Statistical Sciences

バージョン :

権利関係 : 


\title{
DYADIC STATIONARY PROCESSES AND THEIR SPECTRAL REPRESENTATIONS
}

\author{
By
}

\author{
Takeaki NAGAI*
}

(Received October 2, 1976)

\begin{abstract}
A class of random processes whose covariance functions are in. variant under the shift by the dyadic addition is considered. These processes are called to be dyadic stationary.

It is shown in theorem 2 that a dyadic stationary process has always spectral representation in terms of the system of Walsh functions parallel to an ordinary stationary process in terms of the system of trigonometric functions.

By making use of spectral representation in terms of the system of Walsh functions, necessary and sufficient conditions for a dyadic stationary process to be a dyadic linear process introduced in Morettin (1974) are shown in theorems 3 and 4.
\end{abstract}

\section{Preliminaries.}

We denote by $I$ the set of all real numbers in the unit interval [0.1] and by $N$ the set of all non-negative integers.

Let $x$ and $y$ be two non-negative real numbers and have the dyadic expansions:

$$
\begin{aligned}
& x=\sum_{k=-\infty}^{\infty} x_{k} \cdot 2^{k}, \quad \text { with } x_{k}=0 \text { or } 1, \\
& x=\sum_{k=-\infty}^{\infty} y_{k} \cdot 2^{k}, \quad \text { with } y_{k}=0 \text { or } 1 .
\end{aligned}
$$

When the expansion, for instance say (1.1), is not unique, we adopt the expansion in which zeros appear infinitely often for $x_{k}, k<0$.

Then the dyadic addition $\ominus$ is defined by

$$
x \oplus y=\sum_{k=-\infty}^{\infty}\left(x_{k} \oplus y_{k}\right) \cdot 2^{k},
$$

where $x_{k} \oplus y_{k}$ denotes addition mod. 2 of $\{0,1\}$, that is, $0 \oplus 0=1 \oplus 1=0$ and $1 \oplus 0$ $=0 \oplus 1=1$. (Cf. [4], [5].)

AMS 1970 Subject classifications. Primary 60G10, 60B99; Secondary 10K35

Key words and phrases. Dyadic stationary processes, dyadic linear processes, spectral representations, dyadic spectral distribution functions, Walsh functions.

* Faculty of Engineering, Oita University. 
We denote by $\{W(n, x), x \in I\}, n=0,1,2, \cdots$, the system of Walsh functions (WF). (Cf. [4], [5], [6].)

The following properties of the system of WF are well-known:

(i) The system of WF is orthonormal on $I$, that is,

$$
\begin{aligned}
& \int_{0}^{1} W(n, x) W(m, x) d x=1, \text { for } n=m, \\
& =0 \text {, for } n \neq m \text {, }
\end{aligned}
$$

and form a complete set. Thus every square integrable function $f(x)$ on $I$ can be expanded as a Walsh-Fourier series of the form:

$$
f(x)=\sum_{n=0}^{\infty} c(n) W(n, x), \quad \text { a.e. } x \in I,
$$

where $c(n)=\int_{0}^{1} f(x) W(n, x) d x$.

(ii) For any $n, m \in N$ and for a.e. $x \in I$,

$$
W(n, x) W(m, x)=W(n \oplus m, x) .
$$

For each $n \in N$, each $y \in I$ and a.e. $x \in I$,

$$
W(n, x) W(n, y)=W(n, x \oplus y) .
$$

\section{Dyadic stationary processes.}

We call a random process $\{X(n), n \in N\}$ with constant means and finite second moments to be dyadic stationary if its covariance function

$$
R(n, m)=E\{(X(n)-E[X(n)])(X(m)-E[X(m)])\}, n, m \in N,
$$

is invariant under the shift by the dyadic addition $\oplus$, that is, for every $n, m, k \in N$,

$$
R(n, m)=R(n \oplus k, m \oplus k)=R(n \oplus m, 0) .
$$

We assume for simplicity that

$$
\begin{aligned}
& E[X(n)]=0, \quad \text { for all } n \in N, \text { and } \\
& E\left[X(n)^{2}\right]=1, \text { for all } n \in N .
\end{aligned}
$$

We show at first that a covariance function of a dyadic stationary process has spectral representation in terms of the system of WF and thus a dyadic spectral distribution on the sequency domain will be theoretically defined analogously to an ordinary power spectral distribution.

THEOREM 1. Let $\{X(n), n \in N\}$ be a dyadic stationary process satisfying (2.3) and (2.4). Then its covariance function $R(n, m)$ can be represented in the following form:

$$
R(n, m)=\int_{0}^{1} W(n \oplus m, x) d G(x),
$$

where $G(x), x \in I$, is a unique distribution function on $I$.

We call the function $G(x), x \in I$, the dyadic spectral distribution of the process $\{X(n), n \in N\}$. 
Proof. Since the covariance function $R(n, m), n, m \in N$, depends only on $n \oplus m$, we put $R(n)=R(n \oplus m, m), n, m \in N$, for notational conveniency.

We define a sequence of functions $\left\{F_{m}(x), x \in I\right\}, m=0,1,2, \cdots$, by

$$
F_{m}(x)=\sum_{n=0}^{2^{m}-1} R(n) \cdot \int_{0}^{x} W(n, t) d t, x \in I .
$$

It is easily seen that $F_{m}(x), x \in I, m=0,1,2, \cdots$, are distribution functions since for all $m=0,1,2, \cdots, F_{m}(0), F_{m}(1)=1$, and for each $x \in I$, the integrands of $(2.6)$ are non-negative, that is,

$$
\begin{aligned}
\sum_{n=0}^{2_{-1}} R(n) W(n, t) & =2^{-m} \cdot \sum_{j=0}^{2^{m}} \sum_{k=0}^{2^{m}-1} R(j \oplus k) W(j \oplus k, t) \\
& =2^{-m} \cdot E\left[\left(\sum_{j=0}^{m_{-1}} X(j) W(j, t)\right)^{2}\right] \geqq 0 .
\end{aligned}
$$

The sequence $\left\{F_{m}(x), x \in 1\right\}, m=0,1,2, \cdots$, is trivially mass-preserving. (Cf. [2], p. 162.) Hence, it follows from Prohorov's theorem (see [2], p. 162 or [1], p. 37) that there exists a weakly convergent subsequence $\left\{F_{m(j)}(x), x \in I\right\}, j=1,2,3, \cdots$, and a distribution function $G(x), x \in I$, such that

$$
F_{m(j)}(x) \longrightarrow G(x) \text {, in law, as } j \longrightarrow \infty \text {. }
$$

Since the system of WF are uniformly integrable with respect to the family of the distribution functions $\left\{F_{m(j)}(x), x \in I\right\}, j=1,2,3, \cdots$, that is, for every $\varepsilon>0$ and for all $n \in N$,

$$
\sup \int_{0}^{1}|W(n, x)|^{1+\varepsilon} \cdot d F_{m(j)}(x) \leqq 1,
$$

we see that for all $n \in N$,

$$
\int_{0}^{1} W(n, x) d G(x)=\lim _{j \rightarrow \infty} \int_{0}^{1} W(n, x) d F_{m(j)}(x) .
$$

While from the definition of $F_{m}(x)$ we have

$$
\begin{aligned}
\int_{0}^{1} W(n, x) d F_{m}(x) & =R(n), \text { for } n \leqq 2^{m}-1, \\
& =0, \quad \text { for } n>2^{m}-1 .
\end{aligned}
$$

Thus, it follows that for every $n=0,1,2, \cdots$,

$$
\begin{aligned}
R(n) & =\lim _{j \rightarrow \infty} \int_{0}^{1} W(n, x) d F_{m(j)}(x) \\
& =\int_{0}^{1} W(n, x) d G(x) .
\end{aligned}
$$

Suppose now that there are two distribution functions $G(x)$ and $H(x)$ such that

$$
\begin{aligned}
R(n) & =\int_{0}^{1} W(n, x) d G(x) \\
& =\int_{0}^{1} W(n, x) d H(x), \quad \text { for all } n \in N .
\end{aligned}
$$


For an open interval $J=\left(k / 2^{m}, r / 2^{m}\right)$ with dyadic rational end-points, its indicator function $I_{J}(\cdot)$ is expressed as a Walsh-Fourier series of finite terms:

$$
I_{J}(x)=\sum_{n=0}^{M} c_{J}(n) W(n, x), x \in I,
$$

where $M$ is an integer and

$$
c_{J}(n)=\int_{J} W(n, x) d x
$$

Hence, we have from (2.11) $G(J)=\sum_{n=0}^{M} c_{J}(n) R(n)=H(J)$. Since the family of all open intervals with dyadic rational end-points generates the $\sigma$-field of Borel subsets of $I$, we see that for all Borel set $A$ of $I, G(A)=H(A)$.

It is also shown that spectral representation theorem holds for a dyadic stationary process analogously to an ordinary stationary process.

THEOREM 2. Let $\{X(n), n \in N\}$ be a dyadic stationary process satisfysng (2.3) and (2.4) and having a dyadic spectral distribution $G(x), x \in I$.

Then the process $\{X(n), n \in N\}$ has a (dyadic) spectral representation

$$
X(n)=\int_{0}^{1} W(n, x) d Z(x), n \in N,
$$

where $\{Z(x), x \in I\}$ is a real random process with orthogonal increments such that

$$
E\left[(d Z(x))^{2}\right]=d G(x), \quad x \in I .
$$

Proof. Let $L_{2}(X)$ be a Hilbert space consisting of all random variables which may be represented either as a finite linear combination

$$
\xi=\sum_{i=1}^{k} c_{i} X\left(n_{i}\right)
$$

for some integers $n_{1}, n_{2}, \cdots, n_{k} \in N$ and real numbers $c_{1}, c_{2}, \cdots, c_{k}$ or as a limit in quadratic mean of such finite linear combinations under the inner product defined by $(\xi, \eta)=E[\xi \cdot \eta]$. Let $L_{2}(G)$ be a Hilbert space consisting of all square integrable functions on $I$ with respect to $G(x), x \in I$. We write the inner product of each pair of functions $\left(f_{1}, f_{2}\right)$ in $L_{2}(G)$ as

$$
\left(f_{1}, f_{2}\right)_{G}=\int_{0}^{1} f_{1}(x) f_{2}(x) d G(x) .
$$

We assume without loss of generality that the system of WF $\{W(n, x), x \in I\}$, $n=0,1,2, \cdots$, spans $L_{2}(G)$. (Otherwise, by adding to the system of WF a suitably chosen functions $\{\xi(r, x), x \in I\}, r=1,2,3, \cdots$, in $L_{2}(G)$ which are complementarily orthonormal to the system of WF and forming a complete set in $L_{2}(G)$, we can proceed our proof of theorem 2 similarly to the case where the Walsh functions span $L_{2}(G)$.)

Since $L_{2}(X)$ is spanned by $\{X(n), n \in N\}$ and $L_{2}(G)$ by $\{W(n, \cdot), n \in N\}$ and for every $n, m \in N$, 


$$
\begin{aligned}
(W(n, \cdot), W(m, \cdot))_{G} & =\int_{0}^{1} W(n, x) W(m, x) d G(x) \\
& =R(n, m) \\
& =(X(n), X(m)),
\end{aligned}
$$

it follows from the basic congruence theorem (ct. [9], p. 962) that there is a linear one-one mapping $\Psi$ from $L_{2}(G)$ onto $L_{2}(X)$ such that

$$
\Psi(W(n, \cdot))=X(n), \quad n \in N,
$$

and for each pair of functions $\left(f_{1}, f_{2}\right)$ in $L_{2}(G)$,

$$
\left(f_{1}, f_{2}\right)_{G}=\left(\Psi\left(f_{1}\right), \Psi\left(f_{2}\right)\right) \text {. }
$$

We denote by $\mathfrak{B}$ the $\sigma$-field of Borel subsets of $I$.

Let us put

$$
Z(A)=\Psi\left(I_{\boldsymbol{A}}\right) \in L_{2}(X), \text { for } A \in \mathfrak{B} .
$$

Then it is shown from (2.16) that the family of random variables $\{Z(A), A \in \mathfrak{B}\}$ has the following properties:

$$
E[Z(A)]=0, \text { for } A \in \mathfrak{B},
$$

and for every pair of sets $(A, B)$ in $\mathfrak{B}$,

$$
\begin{aligned}
E[Z(A) Z(B)] & =\left(I_{A}, I_{B}\right)_{G} \\
& =G(A \cap B) .
\end{aligned}
$$

In particular, by putting

$$
Z(x)=Z([0, x]), \quad x \in I,
$$

we obtain a real random process $\{Z(x), 0 \leqq x \leqq 1\}$, with orthogonal increments satisfying (2.13).

In order to define the stochastic integral of a function $f \in L_{2}(G)$ with respect to $\{Z(x), 0 \leqq x \leqq 1\}$, we denote the image of $f$ under the mapping $\Psi$ by

$$
\Psi(f)=\int_{0}^{1} f(x) d Z(x),
$$

and call (2.21) the stochastic integral of $f$ with respect to $\{Z(x), x \in I\}$.

Since the class of indicator functions $\left\{I_{A}, A \in \mathfrak{B}\right\}$ spans $L_{2}(G)$, the definition of the stochastic integral (2.21) is equivalent to the usual stochastic integral of $f$ with respect to $\{Z(x), x \in I\}$ in q. m. sense. (Cf. [3], p. 426.)

Thus, from (2.15) and (2.21), we have a spectral representation of $X(n)$ in the following form:

$$
\begin{aligned}
X(n) & =\Psi(W(n, \cdot)) \\
& =\int_{0}^{1} W(n, x) d Z(x) .
\end{aligned}
$$

EXAMPLE 1. Let $\{u(n), n \in N\}$ be an orthogonal sequence of random variables with 


$$
\begin{array}{r}
E[u(n)]=0, \text { for all } n \in N, \text { and } \\
\begin{aligned}
E[u(n) u(m)] & =\sigma^{2}, \quad \text { for } n=m \\
=0, & \text { for } n \neq m .
\end{aligned}
\end{array}
$$

Then, $\{u(n), n \in N\}$ is a dyadic stationary process and has the spectral representation in terms of Walsh functions

$$
u(n)=\int_{0}^{1} W(n, x) d U(x)
$$

where $\{U(x), x \in I\}$ is a process with orthogonal increments such that

$$
E\left[(d U(x))^{2}\right]=\sigma^{2} \cdot d x .
$$

Hence, the dyadic spectral distribution function of $\{u(n), n \in N\}$ is given by

$$
G(x)=\sigma^{2} \cdot x, \quad 0 \leqq x \leqq 1 .
$$

\section{Linear dyadic processes.}

We call a random drocess $\{X(n), n \in N\}$ a linear dyadic process (see Morettin (1974)) if it can be represented by

$$
X(n)=\sum_{k=0}^{\infty} a(k) u(n \oplus k), \quad n \in N,
$$

where $\{u(n), n \in N\}$ is the sequence of example 1 and $a(k), k \in N$, are real numbers such that $\sum_{k=0}^{\infty} a(k)^{2}<\infty$.

We show at first that a linear dyadic process is dyadic stationary. At the same time we consider the expression of its dyadic spectral density function in terms of the Walsh functions.

THEOREM 3. Let $\{X(n), n \in N\}$ be a linear dyadic process of the form (3.1). Then, it is dyadic stationary and has an absolutely continuous dyadic spectral distribution function. Its dyadic spectral density function is given by

$$
g(x)=\sigma^{2} \cdot\left[\sum_{k=0}^{\infty} a(k) W(k, x)\right]^{2}, \quad x \in I .
$$

Proof. Let us put

$$
h(x)=\sum_{k=0}^{\infty} a(k) W(k, x), \quad x \in I .
$$

Then, by means of the spectral representation (2.25) of $u(n)$, we have the (dyadic) spectral representation of $X(n)$ :

$$
\begin{aligned}
X(n) & =\sum_{k=0}^{\infty} a(k) \cdot \int_{0}^{1} W(n \oplus k, x) d U(x) \\
& =\int_{0}^{1} W(n, x) h(x) d U(x) .
\end{aligned}
$$

It follows from (3.4) that $E[X(n)]=0, n \in N$, and the covariance function of $\{X(n), n \in N\}$ is given by 


$$
\begin{aligned}
R(n, m) & =\int_{0}^{1} \int_{0}^{1} W(n, x) W(m, y) h(x) h(y) E[d U(x) d U(y)] \\
& =\sigma^{2} \cdot \int_{0}^{1} W(n, x) W(m, x)[h(x)]^{2} d x \\
& =\int_{0}^{1} W(n \oplus m, x)[\sigma \cdot h(x)]^{2} d x
\end{aligned}
$$

Thus, from (3.5) it is seen that the process $\{X(n), n \in N\}$ is dyadic stationary and its dyadic spectral density function is given by

$$
\begin{aligned}
g(x) & =\sigma^{2} \cdot[h(x)]^{2} \\
& =\sigma^{2} \cdot\left[\sum_{k=0}^{\infty} a(k) W(k, x)\right]^{2} .
\end{aligned}
$$

We have conversely the following theorem.

THEOREM 4. Let $\{X(n), n \in N\}$ be a dyadic stationary process with an absolutely continuous dyadic spectral distribution function. Then, it is a linear dyadic process if its dyadic spectral density function is not zero almost everywhere.

Proof. Denote by $G(x)$ the dyadic spectral distribution function of $\{X(n)$, $n \in N\}$ and by $g(x)=d G(x) / d x$ the dyadic spectral density function.

From theorem 2, $X(n)$ has a spectral representation

$$
X(n)=\int_{0}^{1} W(n, x) d Z(x), \quad n \in N,
$$

where $\{Z(x), x \in I\}$ is a random process with orthogonal increments such that

$$
E\left[(d Z(x))^{2}\right]=d G(x) .
$$

Let us put

$$
L(x)=\int_{0}^{x}[g(t)]^{-1 / 2} d Z(t), \quad x \in I
$$

Then, the random process $\{U(x), x \in I\}$ is well-defined since $g(x)^{-1 / 2}$ is square integrable with respect to $G(x)$. We see easily that the process $\{U(x), x \in I\}$ has orthogonal increments with

$$
E\left[(d U(x))^{2}\right]=d x .
$$

From (3.9), $d Z(x)=g(x)^{1 / 2} d U(x)$. Substuting this relation into (3.7), we have

$$
X(n)=\int_{0}^{1} W(n, x) \cdot[g(x)]^{1 / 2} d U(x) .
$$

The function $g(x)^{1 / 2}$ being square integrable, we can expand $g(x)^{1 / 2}$ as a WalshFourier series

$$
g(x)^{1 / 2}=\sum_{k=0}^{\infty} a(k) W(k, x), \quad x \in I,
$$

with $\sum_{k=0}^{\infty} a(k)^{2}<\infty$. By making use of (3.12), we have the following expansion of $X(n)$ : 


$$
\begin{aligned}
X(n) & =\int_{0}^{1} W(n, x)\left[\sum_{k=1}^{\infty} a(k) W(k, x)\right] d U(x) \\
& =\int_{0}^{1} \sum_{k=0}^{\infty} a(k) W(n \oplus k, x) d U(x) \\
& =\text { 1.i.m } \sum_{k=0}^{M} a(k) \cdot \int_{0}^{1} W(n \oplus k, x) d U(x) \\
& =\sum_{k=0}^{\infty} a(k) u(n \oplus k),
\end{aligned}
$$

where we have put

$$
u(k)=\int_{0}^{1} W(k, x) d U(x), \quad k \in N .
$$

From (3.10), we see that $\{u(n), n \in N\}$ is the same sequence of example 1 with $\sigma^{2}=1$.

Thus, we have proved theorem 4.

Q.E.D.

\section{Concluding remarks.}

By making use of these theorems 2,3 and 4, stochastic characterization problems of dyadic stationary processes will become much easier to treat with. Two of such examples are stated briefly as concluding remarks.

(i) For a time series $\{X(n), n=0,1,2, \cdots, T-1\}$ with length $T=2^{m}$, ( $m$ is a positive integer), as a realization of a random process $\{X(n), n \in N\}$, let us define finite Walsh transforms of the time series $\{X(n), n=0,1,2, \cdots, T=1\}$ by

$$
Y(j)=\sum_{n=0}^{T-1} W(n, x(j)) X(n), \quad j=0,1,2, \cdots, T-1,
$$

where we have put $x(j)=(2 j+1) / 2 T$. (Cf. [7], [8].)

Then, it is shown in Nagai (1976) that for all $m=1,2,3, \cdots$, the finite Walsh transforms $\{Y(j), j=0,1,2, \cdots, T-1\}$ are uncorrelated when and only when the process $\{X(n), n \in N\}$ is dyadic stationary.

In other words, the dyadic stationarity is characterized by the orthogonality of finite Walsh tansforms.

(ii) Let us call a dyadic linear process of the form (3.1) a dyadic moving average process of order $q(\operatorname{DMA}(q))$ if $a(q) \neq 0$ and $a(q+1)=a(q+2)=\cdots=0$. We call a dyadic stationary process $\{X(n), n \in N\}$ a dyadic autoregressive process of order $p(\operatorname{DAR}(p))$ if it satisfies

$$
\sum_{k=0}^{p} b(k) X(n \oplus k)=u(n), \quad n \in N,
$$

where $b(0)=1, b(p) \neq 0$ and $\{u(n), n \in N\}$ is the sequence of example 1 .

Then, using the spectral representation of dyadic stationary processes, we see that if $\{X(n), n \in N\}$ is a $\operatorname{DAR}(p)$-process with $p ; 2^{(m-1)} \leqq p \leqq 2^{m}-1$, then it turns out to be a $\operatorname{DMA}(q)$-process with $q ; q \leqq 2^{m}-1$, and conversely, if $\{X(n), n \in N\}$ is a 
$\operatorname{DMA}\left(q^{\prime}\right)$-process with $q^{\prime} ; 2^{(m-1)} \leqq q^{\prime} \leqq 2^{m}-1$, then it is a $\operatorname{DAR}\left(p^{\prime}\right)$-process with $p^{\prime}$; $p^{\prime} \leqq 2^{m}-1$.

\section{References}

[1] Billingsley, P.: Convergence of Probability Measures, John Wiley 1968.

[2] Breiman, L.: Probability. Addison-Wesley 1968.

[ 3 ] Doob, J.L.: Stochastic Processes. John Wiley 1953.

[4] Gulamhusein, M.N. and Fallside, F.: Short-time spectral and autocorrelation analysis in the Walsh domain. IEEE Trans. Information Theory IT-19 (1973), 615-623.

[ 5 ] Harmuth, H.F.: Transmission of Information by Orthogonal Functions. Springer 1972.

[6] Morettin, P.B.: Walsh function analysis of a certain class of time series. Stochastic Processes and their Applications 2 (1974), 183-193.

[ 7 ] Morettin, P.A.: Estimation of the Walsh spectrum. IEEE Trans. Information Theory IT-22 (1976), 106-107.

[8] NagaI, T.: On the finite Walsh transforms of a dyadic stationary time series. To appear in The Research Reports of Faculty of Engineering, Oita Univ. (1976).

[ 9 ] Parzen, E.: An approach to time series analysis. Ann. Math. Statist. 32 (1961), 951-989. 